\title{
Very intense geomagnetic storms: solar sources, characteristics and cycle distribution
}

\author{
Natalia Szajko ${ }^{1}$, Germán Cristiani ${ }^{1}$, Cristina H. Mandrini ${ }^{1}$ \\ and Alisson Dal Lago ${ }^{2}$ \\ ${ }^{1}$ Instituto de Astronomía y Física del Espacio, Buenos Aires, Argentina \\ email: natisolsz@hotmail.com \\ ${ }^{2}$ Instituto Nacional de Pesquisas Espaciais, São José dos Campos, São Paulo, Brazil
}

\begin{abstract}
We revisit previous studies in which the characteristics of the solar and interplanetary sources of intense geomagnetic storms have been discussed. We consider the very intense geomagnetic storms that occurred during Solar Cycle 23 by setting a value of $\mathrm{Dst}_{\mathrm{min}} \leqslant-200 \mathrm{nT}$ as threshold. We have identified and characterized the solar and interplanetary sources of each storm. After this, we investigate the overall characteristics of the interplanetary (IP) main-phase storm driver, including the time arrival of the shock/disturbance at $1 \mathrm{AU}$, the type of associated IP structure/ejecta, the origin of a prolonged and enhanced southward component $\left(B_{z}\right)$ of the IP field, and other characteristics related to the energy injected into the magnetosphere during the storm.
\end{abstract}

Keywords. Sun: activity, Sun: coronal mass ejections (CMEs), interplanetary medium

\section{Introduction}

It is now well established that major geomagnetic storms are the consequence of a sequence of events that originate in the Sun and result in a geoeffective solar wind flow near Earth (see examples in Brueckner et al. 1998; Webb et al. 2000). Broadly speaking, the geoeffective solar wind disturbances can be separated in two types. One of them is associated to IP coronal mass ejections (ICMEs). ICMEs are the counterparts of CMEs in the IP medium. The other type is associated to the fast solar wind coming from solar coronal holes; this flow interacts with the preceeding slow solar wind in zones called corotating interaction regions (CIRs). Several recent studies have found that major geomagnetic storms may be driven by either ICMEs/MCs or CIRs (see Echer et al. 2008, and references therein).

Solar Cycle 23 is unique in the sense that it is the first of the space age during which the Sun has been imaged almost continuously. The Large Angle and Spectrometric Coronagraph (LASCO, Brueckner et al. 1995), on board the Solar and Heliospheric Observatory (SOHO), has provided a long-term set of CMEs for which several characteristic parameters have been catalogued in a comprehensive data base (http://cdaw.gsf.nasa.gov/CME_list/, Gopalswamy et al. 2009). The combination of LASCO data with observations from other SOHO instruments, such as the Extreme-ultraviolet Imaging Telescope (EIT, Delaboudiniere et al. 1995) and the Michelson Doppler Imager (MDI, Scherrer et al. 1995), allows us to determine the solar CME source region and its magnetic characteristics. In addition to this, the plasma and magnetic field experiments on board the Advanced Composition Explorer (ACE) and Wind give the opportunity of full in situ data coverage in the same period of time.

For each very intense storm $\left(\mathrm{Dst}_{\min } \leqslant-200 \mathrm{nT}\right.$ ) during Cycle 23, we determine the time, angular width and plane-of-sky, expansion and radial velocities of the source CME, 
the type and heliographic location of the CME solar source region (including the characteristics of sunspot groups), and the time duration of the associated flare. After this, we investigate the overall characteristics of the IP main-phase storm driver including the arrival time of the shock/disturbance at $1 \mathrm{AU}$, the type of associated IP structure/ejecta, the origin of a prolonged and enhanced southward component $\left(B_{z}\right)$ of the IP field, and other characteristics related to the energy injected into the magnetosphere during the storm (i.e. the solar wind maximum convected electric field, $E_{y}$ ). Our analysis, thus, complements and extends those of other works in the literature.

\section{Selection criteria of the events}

We use the Dst final values from the World Data Center for Geomagnetism (http://wdc.kugi.kyoto-u.ac.jp/dstdir/index.html) to select the events in our set. The temporal extension of Solar Cycle 23 was taken from October 1996 to December 2008 (see e.g. http://www.ips.gov.au/solar). The hourly averaged Dst data are analyzed and plotted to select storms for which Dst $_{\mathrm{m} \text { in }} \leqslant-200 \mathrm{nT}$. We found 19 cases that comply with our selection criterion and one case for which Dst $_{\mathrm{min}}=-197 \mathrm{nT}$. Taking into account that the data are averaged over one hour, we have decided to include this marginal case in our set. First and second columns of the Table 1 show the date and time when Dst reached its minimum and the corresponding value.

\section{The solar sources: CMEs and their origin at the Sun}

To identify the solar source event and the region from which it originates at the Sun's surface, we have proceeded by tracing back the possible solar candidate from Sun to Earth and, in several ambiguous cases, back from Earth to Sun. Our procedure to identify the solar source event is as follows. We first consider a time window between 24 hours (transit CME speed from Sun to Earth $\approx 1800 \mathrm{~km} \mathrm{~s}^{-1}$ ) and 120 hours (transit CME speed $\approx 350 \mathrm{~km} \mathrm{~s}^{-1}$ ) previous to the geomagnetic event to select a candidate CME. This time window roughly takes into account the range of plausible CME speeds measured in coronagraph data. In order to decrease the possible number of candidates, we first consider only frontside full halo CMEs and, in a second step, partial halo CMEs with a large "apparent" angular width $\left(\mathrm{AW} \geqslant 150^{\circ}\right)$. Once the source CME and the location on the Sun from which it originates are determined, we identify: the AR, the class (in soft $\mathrm{X}$-rays and $\mathrm{H} \alpha$ ) and duration of the associated flare using Solar Geophysical Data reports and GOES data, and the degree of magnetic complexity of the AR using MDI data. The flare duration is taken from the time of impulsive soft X-ray increase to the time when the flux returns either to its pre-flare level or another flare occurrs in a different or the same AR, being clearly distinguishable from the CME associated flare. From the third to the ninth columns of Table 1 we list the AR NOAA number and its heliographic location, the standard sunspot group classification at the time of CME occurrence, the X-ray and $\mathrm{H} \alpha$ classifications for the associated flare, the flare duration computed as discussed above, the time of first appearance in $\mathrm{C} 2$, the CME type, its velocity (second order fitting to $\mathrm{C} 2$ and C3 data), and the CME lateral expansion velocity (computed as discussed in Dal Lago et al., 2003, 2004).

\section{The associated interplanetary medium events}

Since storms are driven by the solar wind magnetic field and plasma impinging on the Earth's magnetosphere, we use here in situ data from instruments aboard ACE to 
Table 1. Very intense geomagnetic storms during Solar Cycle 23 and their solar and IP sources. (1): Low intensity bipolar region where a filament eruption occurs. ${ }^{(2)}$ : Location of AR9393 at the time of occurrence of two CMEs, candidate sources of the two-step storm. ${ }^{(3)}$ : The halo CME associated with this storm is related to the interaction between AR10695 and AR10696. The heliographic coordinates in this column are those of AR10696. (4): This duration corresponds to two consecutives flares in AR10501, one on 18 Nov 2003 - 07:52 UT (M3.2/2N) and the one included in the table. ${ }^{(5)}$ : No LASCO data from July to September 1998. ND in the fifth column means no $\mathrm{H} \alpha$ data.

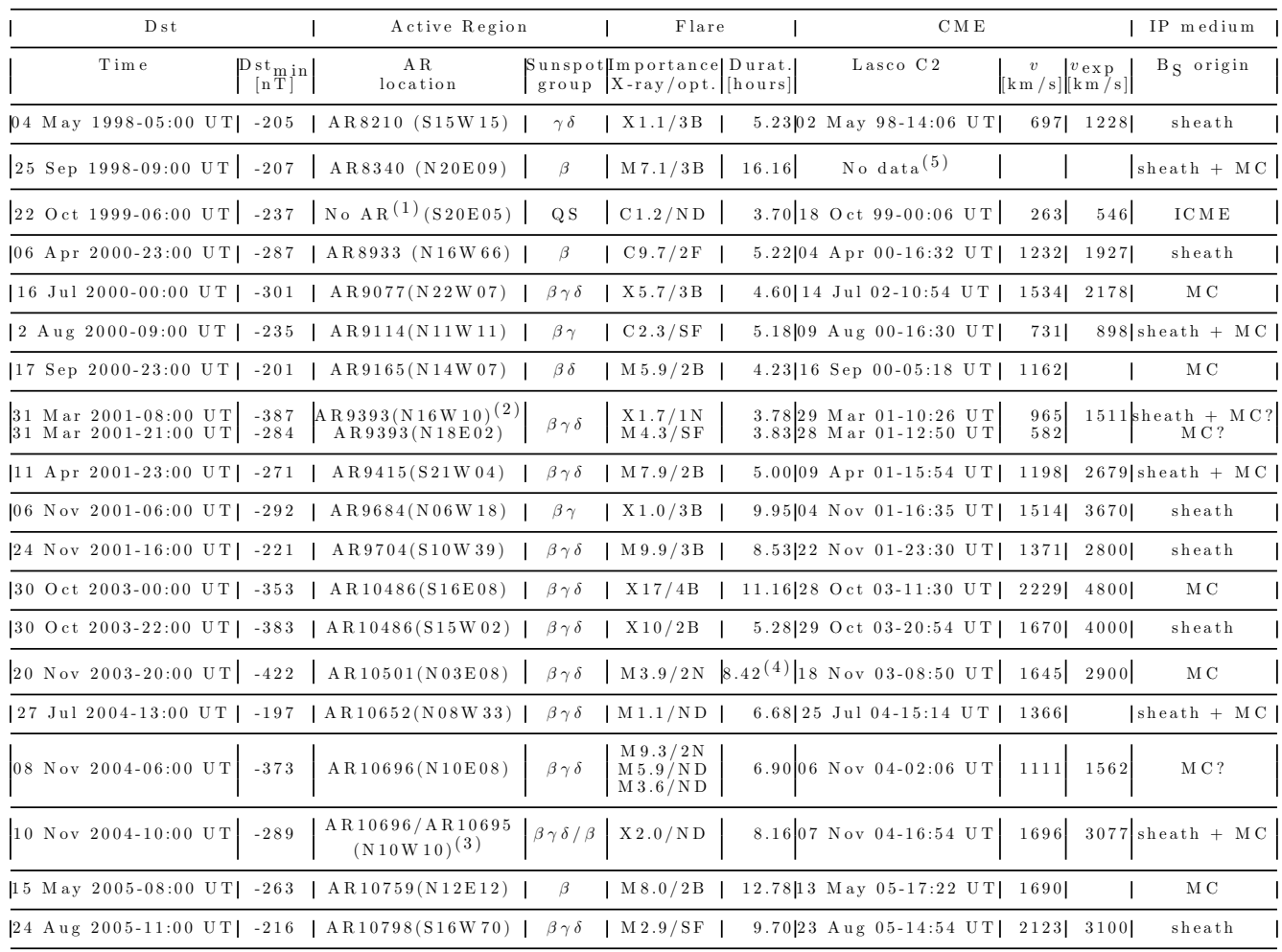

identify the IP structures responsible for each goemagnetic storm. In particular, we have used plasma data from the Solar Wind Electron Proton and Alpha Monitor (SWEPAM, McComas et al. 1998) and magnetic field data from the Magnetic Fields Experiment (MAG, Smith et al. 1998).

Taking into account their magnetic and plasma signatures, we are able to identify various types of structures. These structures include ICMEs, ICMEs containing a flux tube with MC properties, the sheath between the CME driven shock and the ICME, and regions with clear signatures of interaction between ICMEs and high speed streams from coronal holes. Last column of Table 1 indicates the inferred origin of the prolonged and enhanced $B_{z}$.

\section{Summary and discussion}

We have analyzed the full set of very intense geomagnetic storms that occurred during Solar Cycle 23 in search of their solar and interplanetary origin.

When we investigate their distribution, which was double-peaked, we see that $15 \%$ of the events occurs during the cycle rising-phase. All these events are very intense storms. During the first cycle maximum, the number of very intense storms increases to $20 \%$. 
During the second cycle maximum, it reaches $25 \%$ with all of them, but one, being superstorms (following Gonzlez et al. 2002, we call superstorm one for which Dst min $_{\text {in }}$ $\leqslant-250 \mathrm{nT}$ ). However, $40 \%$ of the very intense storms with half of all superstorms (6), including one extreme event, occurs during the cycle descending phase. These results, then, show that the distribution of very intense storms, as that of intense storms along the cycle (see Echer et al. 2008, and references therein), presents two peaks: one during the cycle maximum and another one during the descending phase. These results have been discussed in Gonzalez et al. 2011, and references therein.

All CME sources of the IP disturbances causing very intense geomagnetic storms are either full halo CMEs or partial halos with a large angular width as, in principle, expected. The calculated average lateral expansion velocity of the CMEs is $\approx 2400 \mathrm{~km} \mathrm{~s}^{-1}$. With the lateral expansion velocity, we can apply the phenomenological expression derived by Dal Lago et al. (2003) and compute the Sun-Earth line velocity, in all cases this velocity is much higher than the projected plane-of-the-sky speed. All the listed CMEs, but one, are really fast events with radial velocities reaching $4000 \mathrm{~km} \mathrm{~s}^{-1}$ with an average value of $\approx 2200 \mathrm{~km} \mathrm{~s}^{-1}$. It is also evident from our analysis that all fast CMEs are strongly decelerated during their transit to $1 \mathrm{AU}$, while slow CMEs (see e.g. event number 3) are accelerated by the ambient solar wind.

The CME solar sources of all analyzed storms, but one, are ARs. The ARs where the CMEs originate show, in general, high magnetic complexity; $\delta$ spots are present in $74 \%$ of the cases, $10 \%$ are formed by several bipolar sunspot groups, and only $16 \%$ present a single bipolar sunspot group. This is not surprising as it is well-known that ARs contaning $\delta$ spots display a high level of activity (see Zirin \& Liggett 1987).

All CMEs are associated to long duration events (LDEs), exceeding 3 hours in all cases, with around $75 \%$ lasting more than 5 hours. The associated flares are, in general, intense events, classified as $\mathrm{M}$ or $\mathrm{X}$ in soft X-rays; only three of them fall in the $\mathrm{C}$ class.

When we look for the location of the CME source regions producing very intense storms, we find that $75 \%$ are located at a distance smaller than half a solar radius from the solar disk center (one flare/CME is not observed by SOHO). If we separate this restricted set in Dst $_{\mathrm{m} \text { in }}$ ranges, the ones in the ranges with lower absolute values have source regions at larger distances from Sun center. In four of these cases the AR is closer to the western limb, with two events at a longitude $\geqslant 60^{\circ}$.

Finally, considering the IP structures responsible for a long and enhanced $B_{z}$, we find that $35 \%$ are MCs or ICME fields, 30\% sheath fields, and 30\% combined sheath and MC or ICME fields. Therefore, for this particular set, any of these structures is equally important. We have found no storm originated by CIR fields, only one storm is related to the compression of an ICME by a high speed stream coming from a coronal hole. Strictly speaking, this is not a CIR as it is not a region of interaction between slow and fast solar wind. We have also found that the linear relation between the maximum value of $E_{y}$ and the storm intensity holds (with a correlation coefficient of 0.73 ).

\section{References}

Brueckner, G. E., Delaboudiniere, J.-P., Howard, R. A., Paswaters, S. E., St. Cyr, O. C., Schwenn, R., Lamy, P., Simnett, G. M., Thompson, B., \& Wang, D., 1998, Geophys. Res. Lett., 25, 3019

Brueckner, G. E., Howard, R. A., Koomen, M. J., Korendyke, C. M., Michels, D. J., Moses, J. D., Socker, D. G., Dere, K. P., Lamy, P. L., Llebaria, A., Bout, M. V., Schwenn, R., Simnett, G. M., Bedford, D. K., \& Eyles, C. J. 1995, Sol. Phys., 162, 357

Dal Lago, A., Vieira, L. E. A., Echer, E., Gonzalez, W. D., de Gonzalez, A. L. C., Guarnieri, F. L., Schuch, N. J., \& Schwenn, R. 2004, Sol. Phys., 222, 323 
Dal Lago, A., Schwenn, R., \& Gonzalez, W. D. 2003, Adv. Spac. Res., 32, 2637

Delaboudiniere, J.-P., Artzner, G. E., Brunaud, J., Gabriel, A. H., Hochedez, J. F., Millier, F., Song, X. Y., Au, B., Dere, K. P., Howard, R. A., Kreplin, R., Michels, D. J., Moses, J. D., Defise, J. M., Jamar, C., Rochus, P., Chauvineau, J. P., Marioge, J. P., Catura, R. C., Lemen, J. R., Shing, L., Stern, R. A., Gurman, J. B., Neupert, W. M., Maucherat, A., Clette, F., Cugnon, P., \& van Dessel, E. L. 1995, Sol. Phys., 162, 291

Echer, E., Gonzalez, W. D., Tsurutani, B. T., \& Gonzalez, A. L. C. 2008, Jour. of Geophys. Res. (Space Physics), 113(A12), A05221

Gonzalez, W. D., Tsurutani, B. T., Lepping, R. P., \& Schwenn, R. 2002, Jour. of Atmos. and Solar-Terres. Phys., 64, 173-181

Gopalswamy, N., Yashiro, S., Michalek, G., Stenborg, G., Vourlidas, A., Freeland, S., \& Howard, R., 2009, Earth Moon and Planets, 104, 295

McComas, D. J., Bame, S. J., Barker, P., Feldman, W. C., Phillips, J. L., Riley, P., \& Griffee, J. W. 1998, Space Science Reviews, 86, 563

Scherrer, P. H., Bogart, R. S., Bush, R. I., Hoeksema, J. T., Kosovichev, A. G.,Schou, J., Rosenberg, W., Springer, L., Tarbell, T. D., Title, A., Wolfson, C. J., \& Zayer, I. 1995, Sol. Phys., 162, 129

Smith, C. W., L'Heureux, J., Ness, N. F., Acuña, M. H., Burlaga, L. F., \& Scheifele, J. 1998, Space Scien. Rev., 86, 613

Gonzalez, W. D., Echer, E., Tsurutani, B. T., Clúa de Gonzalez, A. L., \& Dal Lago, A. 2011, Space Science Reviews, 158, 69

Webb, D. F., Lepping, R. P., Burlaga, L. F., DeForest, C. E., Larson, D. E., Martin, S. F., Plunkett, S. P., \& Rust, D. M. 2000, Jour. of Geophys. Res., 105, 27251

Zirin, H. \& Liggett, M. A. 2004, Sol. Phys., 113, 267 